\title{
Photoluminescence and Lifetime Measurement for the Excitation and Temperature Dependence of Carrier Relaxation in III-V Semiconductors
}

\author{
Kathy Kyaw Min ${ }^{1}$, Phyoe Sandar Win ${ }^{1}$, Hla Myo Tun², Zaw Min Naing ${ }^{2}$, Win Khaing Moe \\ ${ }^{1}$ Department of Electronic Engineering, Mandalay Technological University, Mandalay, Myanmar \\ ${ }^{2}$ Department of Electronic Engineering, Yangon Technological University, Yangon, Myanmar
}

Email address:

makathykyawmin.mtu@gmail.com (K. K. Min), phyoesandarwin.mtu@gmail.com (P. S. Win), hlamyotun.ytu@gmail.com (H. M. Tun), drzaw290615@gmail.com (Z. M. Naing), winkmoe@gmail.com (W. K. Moe)

\section{To cite this article:}

Kathy Kyaw Min, Phyoe Sandar Win, Hla Myo Tun, Zaw Min Naing, Win Khaing Moe. Photoluminescence and Lifetime Measurement for the Excitation and Temperature Dependence of Carrier Relaxation in III-V Semiconductors. Optics. Vol. 7, No. 1, 2018 , pp. 38-42.

doi: $10.11648 /$ j.optics.20180701.16

Received: June 24, 2018; Accepted: July 6, 2018; Published: August 2, 2018

\begin{abstract}
Researches in the field of III-V semiconductor photonic devices have initiate applications in a number of disciplines including lighting, optical communications and biomedical engineering. One of the limiting factors for getting better the photonic devices is the carrier relaxation time. This is the time obligatory for energetic carriers to cool to the edge of their particular bands in a bulk semiconductor material, or to the bottom of a well throughout inter- and intra-sub-band spreading in a heterojunction structure. From these lower energy states, they can afterwards recombine radiatively in photonic devices. This study exploited the nonlinear optical practice of frequency up conversion to complete time-resolved luminescence spectroscopy on epitaxial bulk GaAs samples to analyse carrier relaxation times in each as a function of excitation irradiance and temperature of the sample. There is no electrons and defect energy level in the energy curve for p-type samples. In this study, we focus on the recombination process of yellow-luminescence, which causes the decrease in emission efficiency. The variation of yellow-photoluminescence spectrum shape and intensity, which is caused by occupation YL centers by electrons and thermal activation processes of energy level transitions of electrons by phonon collision in GaAs. The measurement model explains the dependence of the PL intensity on excitation intensity, as well as the PL lifetime and its temperature dependence. We demonstrate that time-resolved PL measurements can be used to find the concentrations of free electrons and acceptors contributing to PL in p-type semiconductors.
\end{abstract}

Keywords: Photoluminescence Measurement, Excitation, Temperature Dependence, Carrier Relaxation, III-V Semiconductors

\section{Introduction}

The semiconductor lighting device has swiftly expanded in attractiveness because of its small size, low cost, and its durable nature in 1962. Manufacturing of these devices has fully fledged exponentially since then, driven mainly by proceeds in both communications and data storage technologies. Until in recent times, the majority of these lighting devices operated in the visible to near infrared spectrum because it was comparatively easy to grow the ternary and quaternary III-V compounds obligatory for these devices using GaAs or InP as a substrate. One of the limiting factors of semiconductor materials used in such kind of devices is a restriction known as the carrier relaxation time. This is the time it acquires electrons, having been excited soaring into the conduction band of the semiconductor material, to cool to the bandedge throughout inter- and intra-sub-band spreading. These band-edge carriers are followed by free to recombine radiatively with obtainable holes in the valance band consequential in luminescence. This parameter is significant since the overall efficiency of the laser depends greatly on making carriers available for radiative recombination as speedily as possible. Jang et al. [1] demonstrated that most energetic carriers in the conduction band cool to the bandedge contained by $15-25$ ps of excitation. From readily available, they can recombine with accessible holes producing photons, 
thus causing the material to luminescent. As more carriers cool to the bandedge, more radiative recombination transpires fabricating increased luminescence intensity. Consequently, a direct correlation between the rise in luminescence intensity and the carrier relaxation over time can be contingent.

The theme of carrier relaxation time in QW structures has been beforehand mentioned by Boggesset al. [2] and Gorski [3] even if with contradictory results. Boggess reported a weak indirect dependence of carrier density on rise time while Gorski originate the rise time of the luminescence to augment with carrier density. It has been recommended that one promising reason for this inconsistency could be the entirety number of excited carriers in every experiment, or the carrier density. At elevated carrier densities, carriers could convey their energy to phonons which, consecutively, could communicate their energy back to erstwhile carriers, thus growing the relaxation time. This is recognized as the hot phonon effect. Because there is a direct relationship between excitation power and carrier density, it should be hypothetically potential to scrutinize a minima in luminescence rise times by captivating data at excitation powers throughout the range stated by both Boggess and Gorski. This would stand for the point where the carrier density is at the required threshold for this hot phonon effect to take place.

In order to enumerate the effects of excitation level and temperature on carrier cooling in photonic semiconductor materials, time-resolved photoluminescence spectroscopy was engaged to measure the luminescence rise times in bulk GaAs sample at changing temperatures and excitation levels. Associating the luminescence rise time with carrier cooling time facilitates estimation of the samples for inclination as both the excitation power and sample temperatures were diverse. The range of excitation powers extended those beforehand adressed by Boggess and Gorski in order to attempt to scrutinize an increase in luminescence rise time due to the hot phonon effect.

The rest of the paper is organized with five sections. Section II presents the theory background of recombination process. Section III mentions the governing equation for the analysis. Section IV demonstrates the implementation of the analysis. Section $\mathrm{V}$ discusses on the experimental results for the implemented systems. Section VI pointed out the conclusion of the research works.

\section{Background Theory}

In view of the fact that the purpose of this research is eventually to explore carrier cooling by determining sample photoluminescence, a succinct prologue on the cause of the photoluminescence, the nature of carrier cooling, and the up conversion process is functional.

\subsection{Electron-Hole Couple Configuration and Recombination}

The manoeuvre of largest part photonic devices is based on the formation or annihilation of electron-hole pairs. The electron-hole pair formation process involves elevating an electron in energy from the valance band to the conduction band, by this means departure a hole following in the valance band. These twosomes are fashioned when an energetic particle, incident on a semiconductor, imparts energy as a minimum equal to the bandgap energy to a valance band electron. One scheme of creating electron-hole pairs is throughout photon amalgamation. For this to come about, photons with an energy superior than the bandgap energy are absorbed and convey their energy to the valence band electrons, accordingly supporting them into the conduction band.

It is in addition potential for an electron to misplace energy by recombining with an obtainable hole in the valance band. This characteristically occurs with electrons at the conduction bandedge and can consequence in a radiative transition where the surplus energy is debauched as photons, frequently encompassing energy equal to the bandgap energy specified by $\mathrm{hv}=\varepsilon \mathrm{g}$

Whereh is Planck's constant, vis the frequency of the emitted photon, and egis the bandgap energy. These emanated photons are the source of the luminescence measured in this research. The progression is called photoluminescence (PL) for the reason that the electron-hole pairs were fashioned by absorption of photons.

Whereas the previous conversation was restricted to the radiative recombination of electron-hole pairs, it is also potential for electrons and holes to combine throughout nonradiative transitions. The recombination rate equation that depicts how carrier density will transform over time due to spontaneous emission in a semiconductor can be printed as [4].

$$
-\frac{\mathrm{dn}}{\mathrm{dt}}=\mathrm{A}_{\mathrm{SRH}} \cdot \mathrm{n}+\mathrm{B}_{\mathrm{rad}} \cdot \mathrm{n}^{2}+\mathrm{C}_{\text {Auger }} \cdot \mathrm{n}^{3}
$$

where $\mathrm{n}$ is the carrier density, $\mathrm{A}_{\mathrm{SRH}}$ is the Shockley-Read-Hall coefficient, $B_{\text {rad }}$ is the radiative coefficient, and $C_{\text {Auger }}$ is the Auger coefficient. While the nonradiative Shockley-Read-Hall and Auger recombination mechanisms were not measured in this research, they have been beforehand examined and addressed in-depth by erstwhile folks [4-6].

\subsection{Electron Spreading in Semiconductor Materials}

In order to initiate the perception of carrier cooling, it is original required to thrash out the fundamental physics behind electron motion in the conduction band. Felix Bloch demonstrated quantum mechanically that as a consequence of the wave nature of electrons, they can move unconstrained throughout a flawlessly well-organized lattice of ions [7]. This inferred that electrons in the conduction band of a perfect (infinite) crystal could stay behind there for an indefinite period. Gr" uneisen afterwards utilized electron transport theory to estimate the spreading probabilities of free electrons transfering in a lattice of ions vibrating according to the Debye theory of lattice heat capacity [8]. He attained an appearance for the temperature dependence of the spreading probability which goes as

$$
\frac{1}{\tau_{\text {lat }}}
$$


Where $\tau_{\text {lat }}$ is the mean time between collisions of electrons with the lattice ions. This does not in attendance the complete depiction, on the other hand, for the reason that it was derived with asupposition of aideal lattice. Abnormalities in the lattice givego up to a second cause for electron scattering. These contain interstitial atoms and impurities, crystal dislocations, and grain boundaries. These Abnormalities will affix to the number of collisions contained by the crystal lattice per unit time. Since they are possessions of the semiconductor sample, on the other hand, they are fundamentally self-governing of the temperature. The spreading prospect is inversely proportional to the mean free time between collisions, so the mean free time between collisions for all contributions to the spreading process can be written as

$$
\frac{1}{\tau}=\frac{1}{\tau_{\text {lat }}}+\frac{1}{\tau_{\text {imp }}}
$$

Where $\tau_{\text {imp }}$ is the mean free time between collisions due to all lattice impurities. As well as these two carrier-scattering mechanisms, there are erstwhile sources of spreading in a real crystal. The progression of alloying in semiconductors results in a random placing of the surrogating atom species in the crystal lattice. This reasons a perturbation of the periodic potential of the lattice, which apparent itself as an additional spreading mechanism for carriers known as alloy spreading [9]. This spreading process is prevailing in alloyed semiconductors.

\subsubsection{Electron-Phonon Interface}

As beforehand assured, it is attractive for electrons, or carriers, at the conduction bandedge to lose energy throughout radiative recombination. The Pauli Exclusion Principle, nevertheless, initiates the obligation that not all of the electrons can survive concurrently at the energy level related with the conduction bandedge. This means that electrons will be disseminated in the conduction band of a semiconductor as distinct by Fermi-Dirac figures. Exhilarating a semiconductor with a laser pulse generates a nonequilibrium condition, ensuing in a carrier temperature higher than the lattice temperature.

Primarily, carrier-carrier spreading dominates, as energy is swapped between the carriers fashioned by the pulse. The carrier-carrier spreading speedily disperses as they attain an ordinary temperature [10]. As time growth, the progression of electron-phonon spreading persists to cool carriers due to the lattice vibrations. In these communications, the surplus energy is conveyed to phonons and dissipated as heat in the lattice. Accordingly, the carriers continue to cool and their temperature ultimately approaches the lattice temperature [11]. Electrons before now at the bandedge continue to recombine both radiatively and nonradiatively throughout the progression conferred in equation 2, fashioning vacant energy levels for the cooling carriers. This practice is demonstrated above in Figure 1 , and the finish outcome is that the progress of the carrier temperature in a semiconductor is intimately tied to the rise in the luminescence signal.

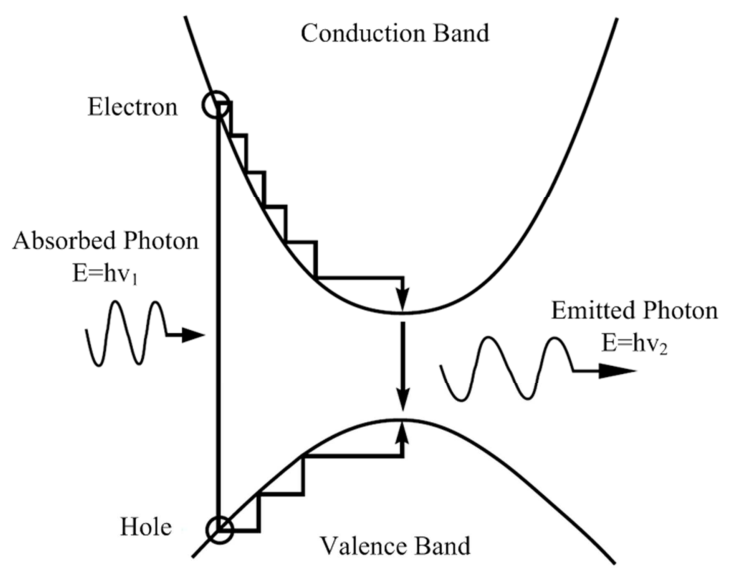

Figure 1. Schematic Representation of Carrier Relaxation in a Semiconductor.

\subsubsection{Hot Phonon Consequence}

An exciting phenomenon that comes about at very highexcitation powers is notorious as the hot phonon effect. At elevated carrier densities, a nonequilibrium phonon population come up as carrier cooling generates phonons more rapidly than the phonon energy can be disseminated, fashioning hot phonons [10]. Energy conveyed to phonons from electrons may possibly, consecutively, be conveyed back to other electrons, thus escalating the relaxation time. This hot phonon effect has been addressed and mentioned by Yang [11].

\subsection{Carrier Cooling in Quantum Wells Structure}

The precedingconversationconcerned the common case, connecting a bulk semiconductor. Photoluminescenceon the other hand can also be calculated in QW structures. In quantum wells structure, the electron-hole population is normally fashioned in the barriers, from which they disperse and thermalize to the lowest bound states in the well region. From convenient, they are capable to recombine radiatively as beforehandillustrated. There are momentousdifferentiations that happen with carrier cooling in QW structures, conversely. It has been in the past reported by Davis that the panorama of a transition between discrete electron levels in a quantum well is much smaller than the probability of making a transition in bulk semiconductor due to energy and momentum conservation requests. This results in a restricted access of carriers in the bulk-like barrier region to the restrained quantum well states as they lingered for the transition to the bottom of the well to crop up. This restricted accessoutcome was appreciably more prominent with narrowing well widths. The narrower the well, the less to be expected the transition will occur, and an increase in carrier relaxation time was practical [12].

\section{Processes to Determine the Non-radiative Recombination Rate}

The emission process of electrons is changed while emitting energy from an excited state to the ground state because of heat.

It referred to as a non-radiative recombination process. Nonradiative recombination is as luminous efficiency reduction 
factor of the optical semiconductor device, each study have been made for the range of semiconductor. This work focuses on the non-radiative recombination process which was the luminous efficiency reduction factor, non-radiative recombination through the deep defect level Bond, but it is by radiative transitions and Auger recombination, via a deep defect level [13-14]. Figure 2 shows the schematic representation of a non-radiative recombination process.

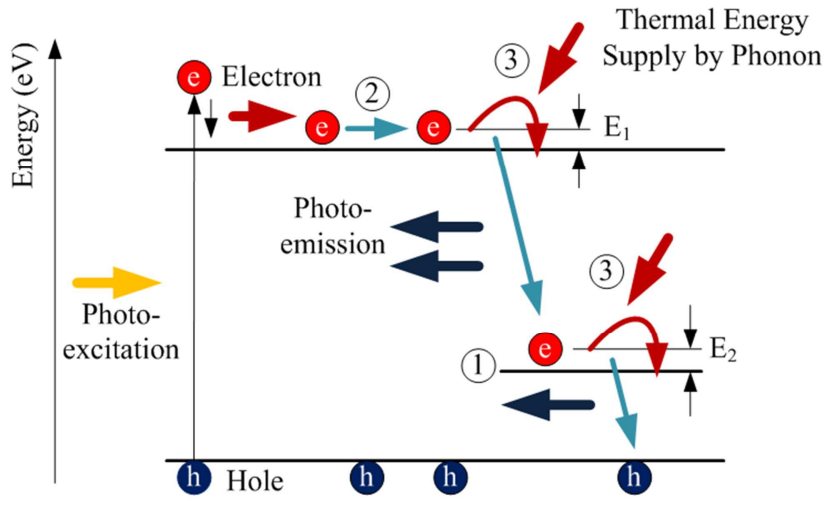

Figure 2. Schematic Representation of a Non-radiative Recombination Process.

\section{Analysis}

At the moment, all of the essentialfoundation has been arranged. The theories following the research and its physical functioning have been described. The samples that were examined have been illustrated. At this moment it is time to pore over the data, beginning with the investigationpractice.

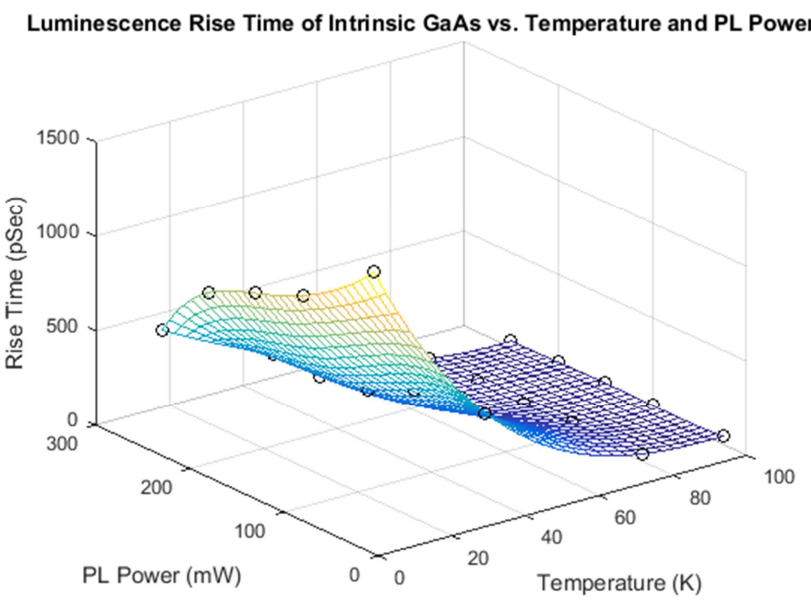

Figure 3. Bulk GaAs rise time as a function of excitation power and temperature. Bicubic interpolation was used to smooth data.

Modern epitaxial growth techniques facilitate very pure binary III-V semiconductor materials such as GaAs to be fashioned. This purity leads to carrier cooling principally throughout electron-phonon communications due to lattice scattering and is evidently demonstrated in Figure 3. It can be observed that the luminescence rise time decreases in cooperation with increased temperature and increased excitation power. The experiential inverse relationship between the temperature and the carrier cooling time was given explanation beforehand. Lattice vibrations increase at the same time as the sample temperature increases, consequently causing increased scattering and lower PL rise times. This in addition explains the decrease in rise time from the increased excitation power additionally. At elevated excitation powers, carrier-carrier spreading originally dominates, but only until they reach an ordinary temperature and in isolation, contribute little to carrier cooling in the lattice [10]. In view of the fact that spreading from impurities is fundamentally constant and GaAs in not an alloy semiconductor, the only mechanism left is spreading from lattice vibrations. The inverse connection between the excitation power and the luminescence rise time was also more obvious at lower excitation powers for the appraised range of temperatures. This is of scrupulous curiosity because it supplementary strengthens the quarrel that excitation power is distressing the sample temperature. As a ultimate hypothesis to the dispute, the supervised temperature of the thermocouple mounted to the cold finger was experiential to raise by less than $2 \mathrm{~K}$ at the amalgamation of the lowest temperatures and the highest excitation powers. This would also point towards that whilst the monitored temperature did not become visible to change at lower excitation powers, there would unmoving be some heating due to the sample irradiance.

\section{Carrier Distribution}

Having established the distribution of available band states and the probability of filling those states under equilibrium conditions, we can now easily deduce the distribution of carriers in the respective energy bands. To be specific, the desired distribution is obtained by simply multiplying the appropriate density of states by the appropriate occupancy factor $-g_{c}(E) f(E)$ yields the distribution of electrons in the conduction band and $g_{v}(E)[1-f(E)]$ yields the distribution of holes in the valence band. GaAs sample carrier distributions for Fermi Energy are pictured in Figure 4 and 5 for the distribution of electrons and distribution of holes. The information just presented concerning the carrier distributions and the relative magnitudes of the carrier numbers finds widespread usage in heterojunction GaAs structure.

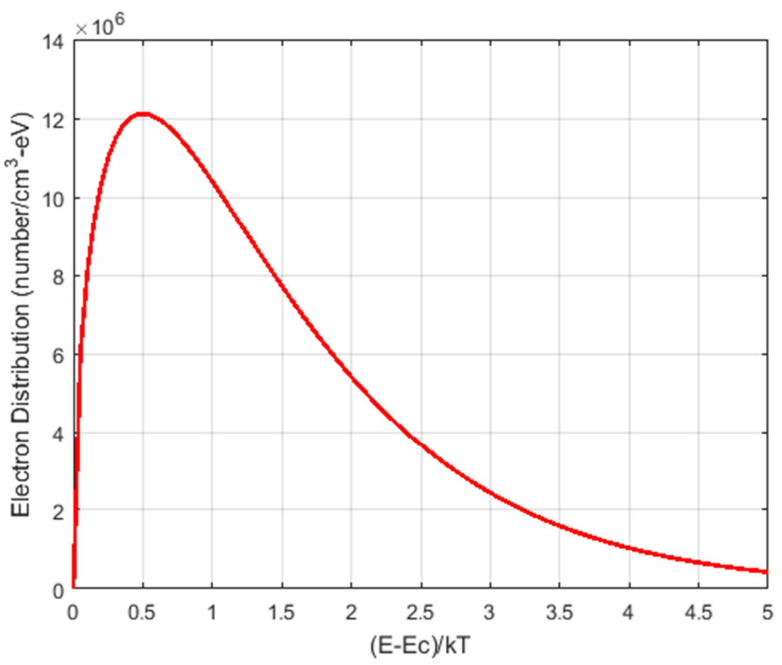

Figure 4. Results of the Electron Distribution. 


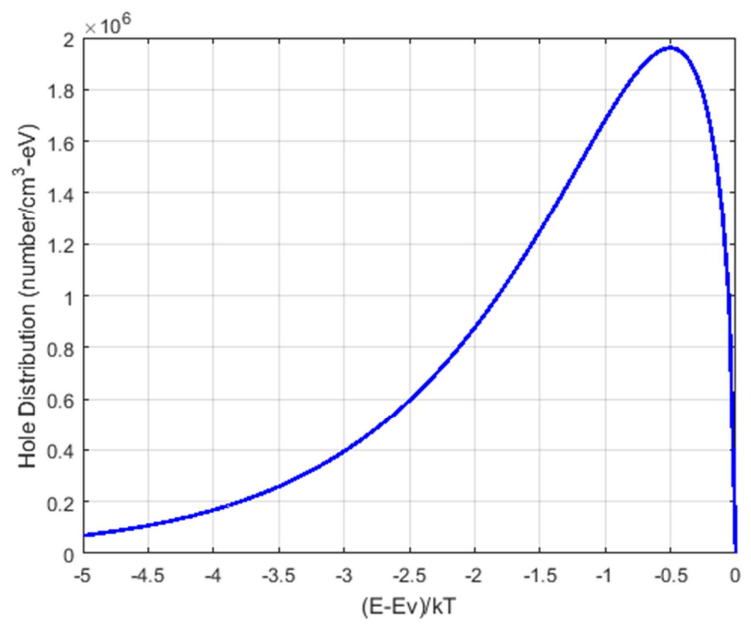

Figure 5. Results of the Hole Distribution.

\section{Lifetime Analysis}

Figure 6 describes the results of the excitation intensity dependent measurement at peak point by $\mathrm{N}_{2}$ laser. We can only observe the slow emission of relaxation; it is a remarkable low energy side, similar relaxation for GaAs sample.

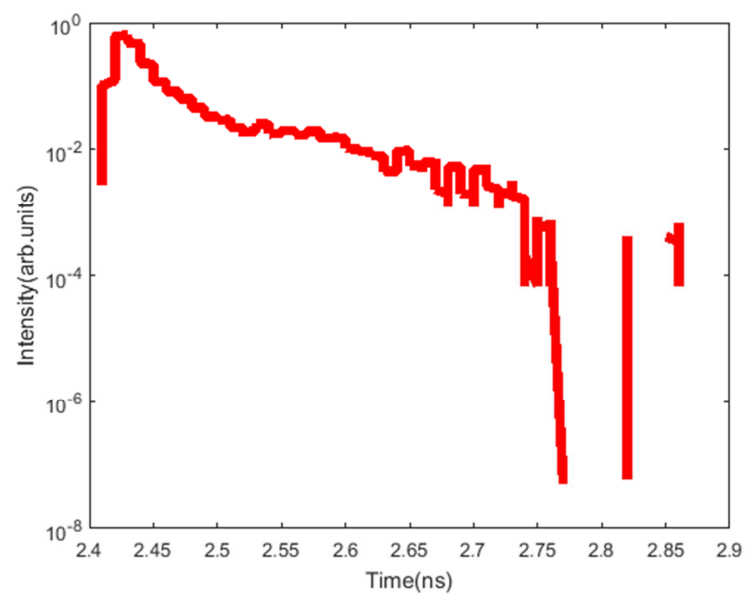

Figure 6. Results of the Lifetime Analysis.

\section{Conclusions}

The implemented results from the bulk GaAssample were the most obvious and, consequently, the easiest to analyze and the mainlyconstructive for future research work. The results obtained from heterojunction structures were a smaller amountunderstandablethough some common trend information was gleaned. With reference to trying to resolve the inconsistencydeclared above, there was still too much variation in the investigational results to arrive at anultimaterespond. It has been demonstrated that the defect-related PL intensity in GaAs. The differences in these dependencies can be explained by the time dependence of the concentration of free electrons, or, otherwise, should be attributed to the time dependencies of the carrier-capture coefficients. The characteristic PL life-times and the lifetime of holes in the valence band can be predicted using a measurement model. The same model explains well the dependence of PL intensity on excitation intensity in time- resolved PL experiments. Analysis of this dependence allows us to find the concentrations of defects that participate in PL.

\section{Acknowledgements}

The author would like to think many colleagues from the Semiconductor Electronics Research Group of the Department of Electronic Engineering of Yangon Technological University.

\section{References}

[1] Jang, D. J., et al. "Hot carrier dynamics in a (GaInSb/InAs)/GaInAlAsSb superlattice multiple quantum well measured with mid-wave infrared, subpicosecond photoluminescence upconversion," Applied Physics Letters, 70 (9):1125-27 (March 1997).

[2] Boggess, Thomas F., et al., "Ultrafast Optical Measurements of Carrier Dynamics in Antimonide-Based Quantum Wells." Thirteenth Annual Solid State and Diode Laser Technology Review, June 5-8 2000.

[3] Gorski, Steven M. Carrier Dynamics in Mid-Infrared Quantum Well Lasers Using Time-Resolved Photoluminescence. MS thesis, Air Force Institute of Technology, Wright-Patterson AFB, OH, March 2002.

[4] Agrawal, A. R. and N. K. Dutta. Semiconductor Lasers (2nd Edition). New York: ITP Van Nostrand Reinhold, 1993.

[5] Cooley, W. T. Measurement of Ultrafast Carrier Recombination Dynamics in Mid-Infrared Semiconductor Laser Material. PhD dissertation, Air Force Institute of Technology, Wright-Patterson AFB, OH, December 1997.

[6] Johnson, Peter M. Deviation of Time-Resolved Luminescence Dynamics in MWIR Semiconductor Materials from Carrier Recombination Theory Predictions. MS thesis, Air Force Institute of Technology, Wright-Patterson AFB, OH, March 2004.

[7] McKelvey, J. P. Solid State Physics for Engineering and Materials Science. Malabar Florida: Krieger Publishing Company, 1993.

[8] Brown, Ronald F. Solid State Physics: An Introduction for Scientists and Engineers. San Luis Obispo, CA: El Corral Publications, 1999.

[9] Bhattacharya, Pallab. Semiconductor Optoelectronic Devices (Second Edition). Prentice Hall, 1997.

[10] Shah, Jagdeep. Ultrafast Spectroscopy of Semiconductors and Semiconductor Nanostructures. Berlin: Springer, 1996.

[11] Yang, C. H., et al. "Hot electron Relaxation in GaAs QuantumWells," Physical Review Letters, 55 (21):2359-2361 (November 1985).

[12] Davis, L., et al. "Carrier Capture and Relaxation in Narrow Quantum Wells," IEEE Journal of Quantum Electronics, 30:2560 (1994).

[13] VadirajKalyaTulasidas, "Photoluminescence and applications of $\mathrm{Ni}$ : ZnS in photovoltaic cells", Japanese Journal of Applied Physics 57, 052302 (2018).

[14] Svetlana V. Boriskina, "Efficiency Limits of Solar Energy Harvesting via Internal Photoemission in Carbon Materials", 5, 4; doi:10. 3390/photonics5010004, Photonics 2018. 